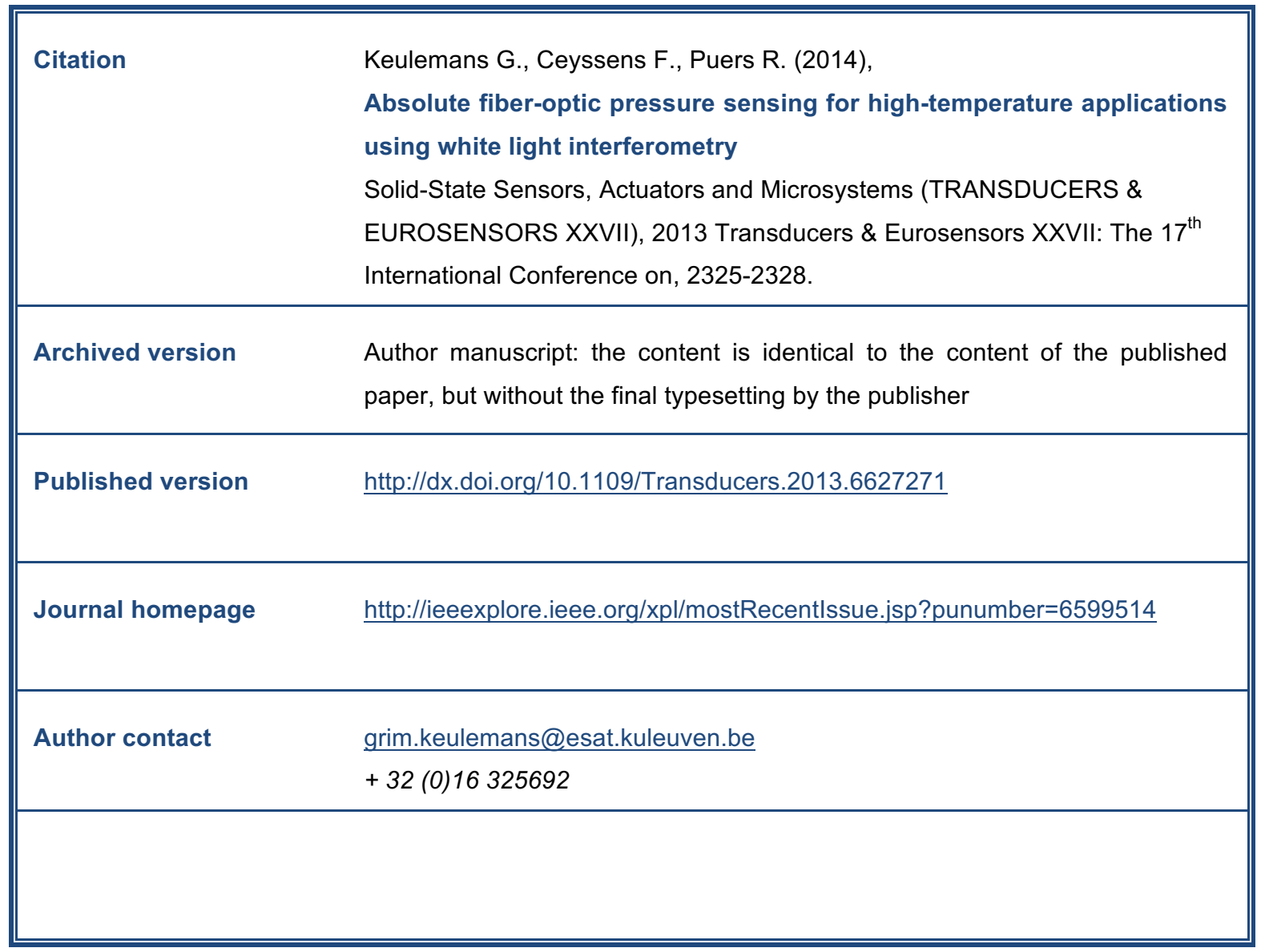




\title{
ABSOLUTE FIBER-OPTIC PRESSURE SENSING FOR HIGH-TEMPERATURE APPLICATIONS USING WHITE LIGHT INTERFEROMETRY
}

\author{
G. Keulemans, F. Ceyssens, and R. Puers \\ Katholieke Universiteit Leuven, Leuven, BELGIUM
}

\begin{abstract}
Fiber-optic pressure sensors allow rugged hightemperature and ultra-miniature pressure monitoring in harsh environments. However, simple light power based readout suffers from bending losses and light source fluctuations. This paper presents a differential technique based on white light interferometry to alleviate this problem. White light spectroscopic measurements have been performed on absolute Fabry-Pérot pressure sensors fabricated at the tip of multi-mode and single mode optical fibers. Bending loss sensitivity is demonstrated to be reduced with a factor of $79 \%$ by using the proposed differential spectroscopic technique.
\end{abstract}

\section{KEYWORDS}

Fiber-optic pressure sensor; focused ion beam; FabryPérot interferometry; white light interferometry

\section{INTRODUCTION}

Fiber-optic sensors are attractive for pressure monitoring. Advantages are their small size (measurements in limited space), their good sensitivity, their harsh environment resilience (electromagnetic interference, corrosive gases ...) and their high operational temperature. Both the pressure sensing membrane and the optical fiber can be made out of one single material, typically silica $[1,2,3]$ or sapphire $[4,5]$. This monolithic nature minimizes critical thermal stresses at elevated temperatures.

Fabry-Pérot (FP) interferometry is used to detect the deflection of a pressure sensitive diaphragm at the tip of an optical fiber. This pressure related deflection will lead to a change in the length of the FP cavity formed between the thin film diaphragm and the end of the optical fiber [1]. As a result, the interference between light which is reflected directly at the fiber end and light which is reflected at diaphragm side of the FP cavity will be altered.

In our research group, several ultra-miniature pressure sensors fabricated directly at the tip of single mode (SM) and multi-mode (MM) optical fibers have been developed [1] (Fig.1.a). More recently an adaption dedicated to fluid dynamic measurements in turbo-machinery has been realized by micro-fabricating the pressure sensitive FP cavity on the sidewall of the optical fiber as shown in Fig.1.b [6]. Operation in high temperature environments up to $600{ }^{\circ} \mathrm{C}$ has been demonstrated [1].

Though the results for these optic-fiber pressure sensors are promising, errors and noise caused by bending of the optical fiber and output fluctuations of the light source still jeopardize reliability. In this paper, a differential technique based on white light interferometry
$[2,3,4,5]$ is introduced to alleviate this problem. Low coherence white light is coupled into the optical fiber. As the length of the FP cavity is still an order of magnitude smaller than the coherence length of the incident light, the reflected light will be modulated by the FP interferometer part of the sensor. A spectrometer is used to detect the changing interference characteristics of the sensor.

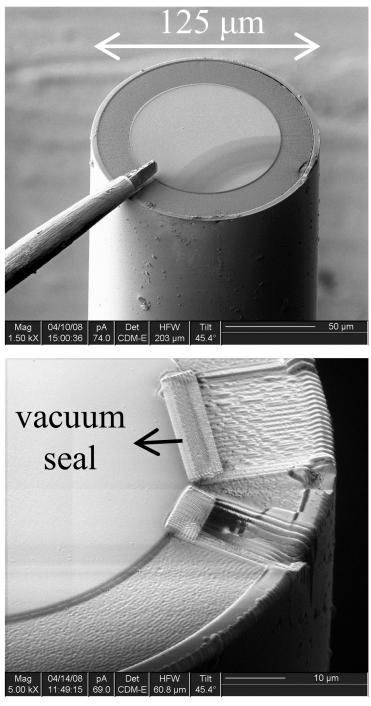

(a)
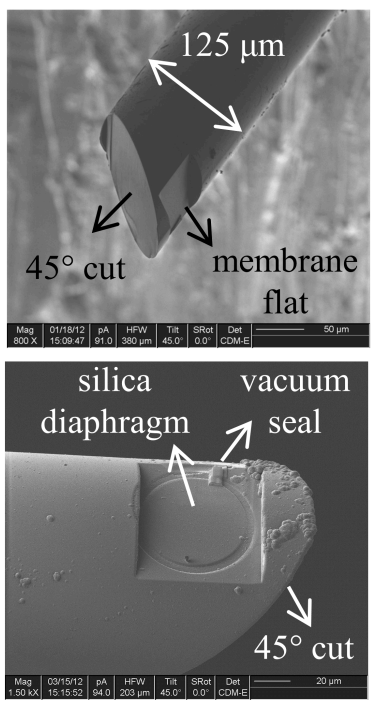

(b)
Figure 1: FIB images of (a) a sealed pressure sensor fabricated at the tip of an optical fiber and $(b)$ an absolute pressure sensor fabricated on the sidewall of an optical fiber optimized for fluid dynamic measurements

First the micro-fabrication process will be addressed, followed by a discussion of the experimental setup and the performed opto-mechanical tests. Measurements have been performed both on MM and SM optical sensors to investigate the potential of using white light interferometry for bending loss compensation.

\section{FABRICATION}

The fabrication process starts with the cleavage and polishing of the end face of an optical fiber (diameter = $125 \mu \mathrm{m})$. Thereafter, FIB machining and thin film deposition steps are used to micro-machine a multilayer FP cavity at the tip of the optical fiber. Molybdenum is used as sacrificial layer. The pressure sensitive diaphragm consists of one or several sputter deposited silica thin films and a metal reflector (Mo or $\mathrm{Cr}$ ). A more detailed description of the fabrication process can be found in [1].

\section{EXPERIMENTAL}

Two measurement setups have been applied to characterize the opto-mechanical operation of the fiberoptic sensors under test. 


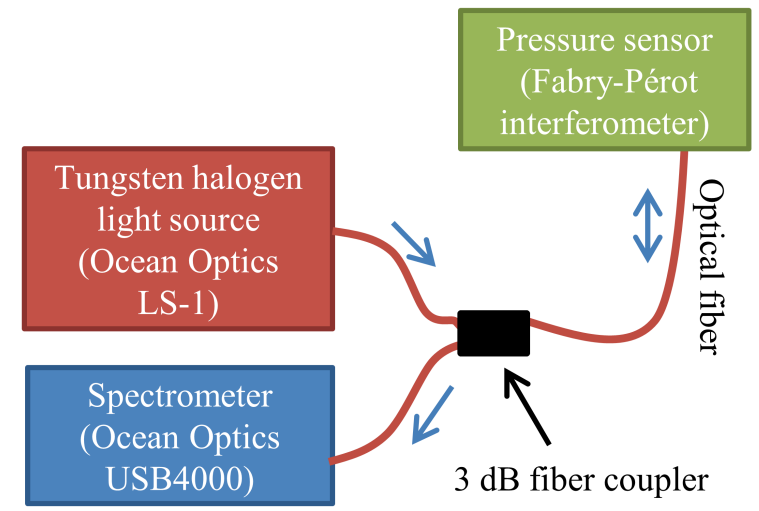

Figure 2: Schematic representation of the white light interferometer measurement setup

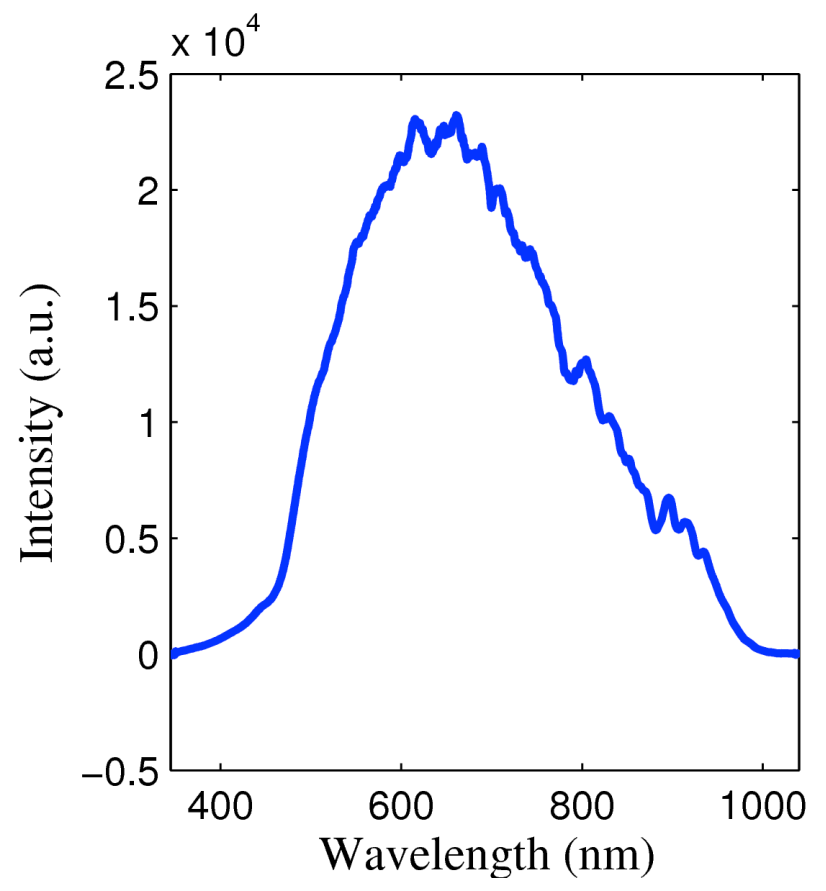

Figure 3: Spectral output of the Ocean Optics LS-1 tungsten halogen light source

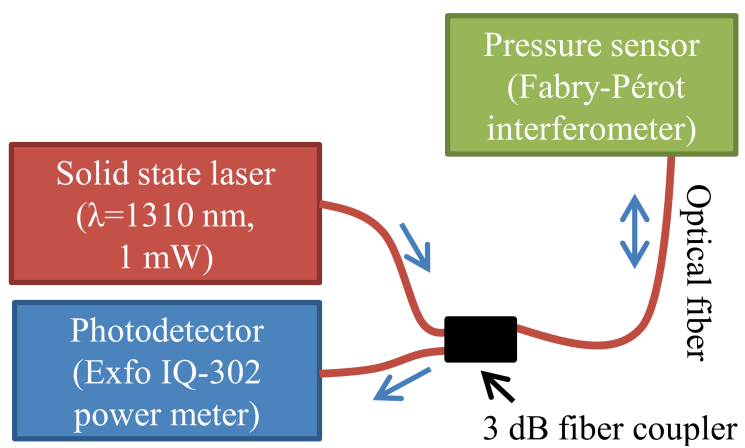

Figure 4: Schematic representation of the solid state laser measurement setup used for SM fiber-optic sensors

White light interferometry measurements have been performed both on MM and SM versions of the developed FP pressure sensors. The setup consists of a broadband tungsten halogen light source (Ocean Optics LS-1), a VIS-NIR spectrometer (Ocean Optics USB4000) and a
$3 \mathrm{~dB}$ fiber coupler (see Fig. 2). The spectral output of the Ocean Optics LS-1 light source is plotted in Fig. 3.

In the case of the SM fiber-optic sensors, the pressure related dependence of the total reflected power is also investigated using a laser based setup (see Fig. 4). This setup includes a $1 \mathrm{~mW}$ solid state laser $(\lambda=1310 \mathrm{~nm})$, a single mode beam splitter and an optical power meter (Exfo IQ-203).

All pressure sensors under test are placed in a pressure controlled chamber and connected to the fiber coupler via an optical feed through. A reference pressure sensor (Druck DPI 104) is present for calibration purposes.

To investigate the potential of white light interferometry in compensating the variations due to bending, different loss factors have been introduced. In the measurement setup, the MM optical fiber that connects to the FP pressure sensor is bent around cylindrical objects of different diameters. An overview of the fiber bending experiments is presented in Table 1. For each bending loss experiment, the spectral dependency with respect to pressure is characterized by increasing the pressure inside the pressure controlled chamber in steps of 250 mbar up to a relative pressure of 2 bar. The measurement results discussed in the following section are gathered using a FP pressure sensor fabricated at the tip of a MM fiber patch cable (OZ Optics MMJ-3S3SIRVIS-62.5/125-3-3). The FP cavity is constructed to have an optical path length of $560 \mathrm{~nm}$ in the unloaded condition. The pressure sensitive diaphragm is composed of a silica-molybdenum-silica multilayer with silica thicknesses of $2080 \mathrm{~nm}$ and $70 \mathrm{~nm}$ respectively and a thickness of $50 \mathrm{~nm}$ for Mo.

Table 1: Measured bending loss cases using a multi-mode fiber sensor

\begin{tabular}{|c|c|}
\hline $\boldsymbol{\emptyset}_{\text {turn }}[\mathbf{m m}]$ & \# turns \\
\hline 155 & 4 \\
\hline 105 & 6 \\
\hline 90 & 7 \\
\hline 65 & 9 \\
\hline
\end{tabular}

Finally the feasibility of white light interferometry in the case of SM optic-fiber sensors is investigated. The measurements results reviewed in the following section are captured using a FP sensor fabricated at the tip of a SM low bending loss patch cable (Thorlabs CCC1310-J9) with a FP length of $890 \mathrm{~nm}$ in the unloaded condition and a multilayer silica-molybdenum-silica diaphragm with silica thicknesses of $1570 \mathrm{~nm}$ and $50 \mathrm{~nm}$ respectively and a thickness of $60 \mathrm{~nm}$ for Mo. The FP sensor design is optimized for highest sensitivity at a central wavelength of $1310 \mathrm{~nm}$.

\section{RESULTS AND DISCUSSION}

In Fig. 5, the white-light interference spectra of a MM optical fiber sensor measured at increasing pressure levels $\left(\varnothing_{\text {turn }}=155 \mathrm{~mm}\right)$ are depicted. Interference peaks and valleys corresponding to constructive and destructive interference as a result of the FP cavity can clearly be 
distinguished, and are superimposed on the spectrum of the tungsten halogen light source. When pressure is applied, the membrane will deflect, thereby decreasing the length of the FP cavity. This results in a shift of the spectral extrema to lower wavelengths. Also a decrease in intensity of the peak regions and an increase in intensity of the valley regions in the range from $500 \mathrm{~nm}$ to $800 \mathrm{~nm}$ are observed.

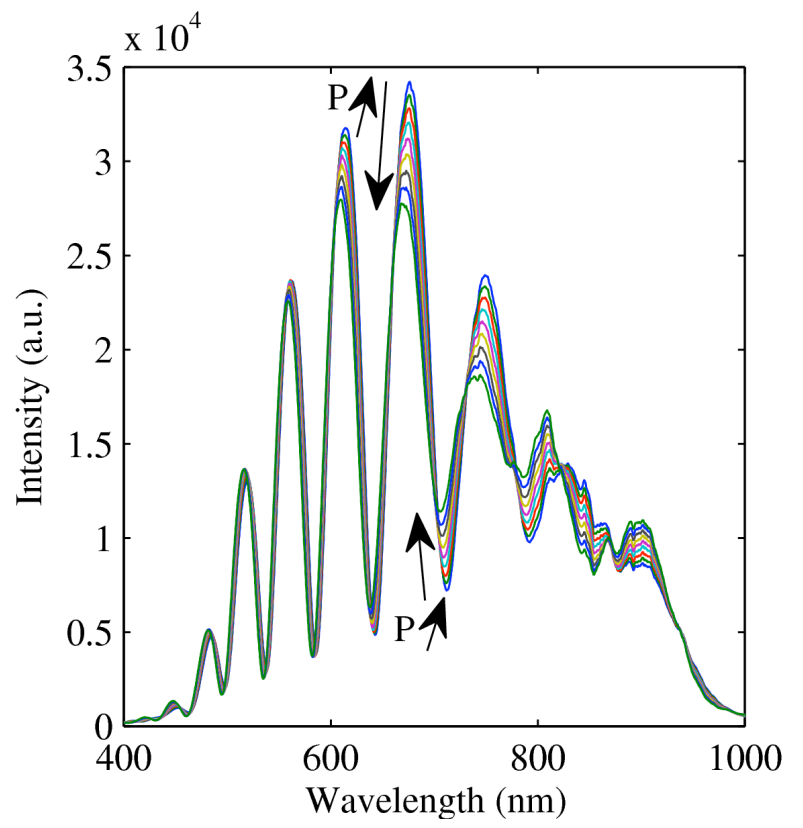

Figure 5: White light interference spectra of a multi-mode fiber sensor with pressure increasing from atmospheric pressure to 2 bar overpressure in steps of $250 \mathrm{mbar}$ $\left(\varnothing_{\text {turn }}=155 \mathrm{~mm}\right)$

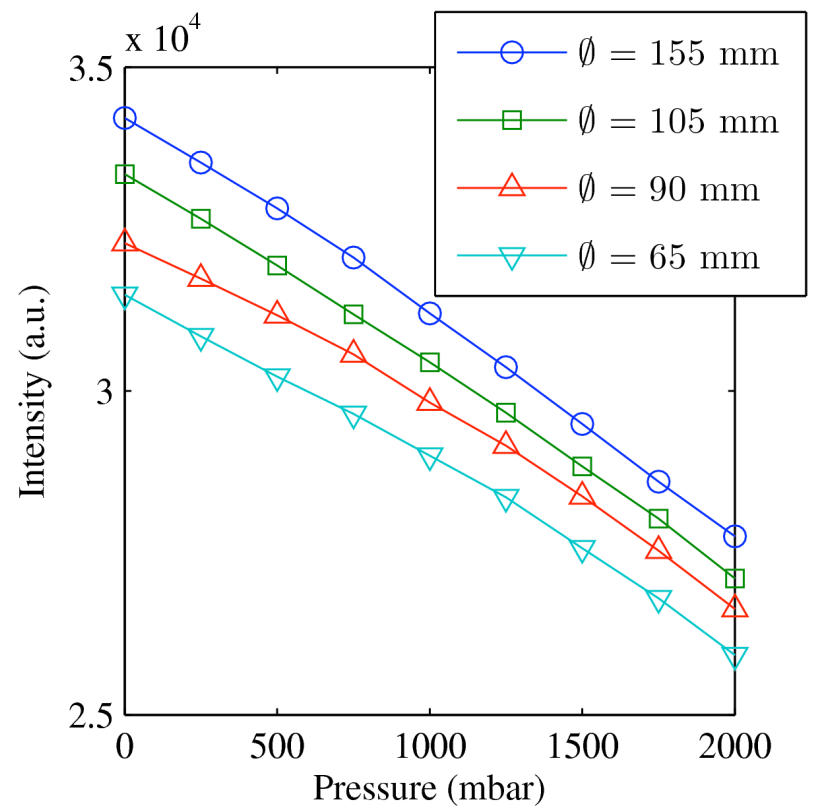

Figure 6: Variation of the intensity of the interference peak at $\lambda=670 \mathrm{~nm}$ as function of relative pressure and different bending losses

Based on the measured spectra, the interference peak at $670 \mathrm{~nm}$ and the valley at $700 \mathrm{~nm}$ have been selected for further analysis. As illustrated in Fig. 6, the single peak intensity value at $670 \mathrm{~nm}$ is quite sensitive to variations in bending loss and light source fluctuations (bending induced measurement error of $33 \%$ full scale (FS)). A similar bending loss dependency is observed for the valley region at $700 \mathrm{~nm}$.

This bending loss dependency can however be compensated for by taking the intensity ratio between a peak (e.g. $670 \mathrm{~nm}$ ) and a nearby valley (e.g. $700 \mathrm{~nm}$ ) as indicated in Fig. 7. By employing this differential technique, the measurement error due to fiber bending could be reduced from $33 \%$ to $7 \%$ FS.

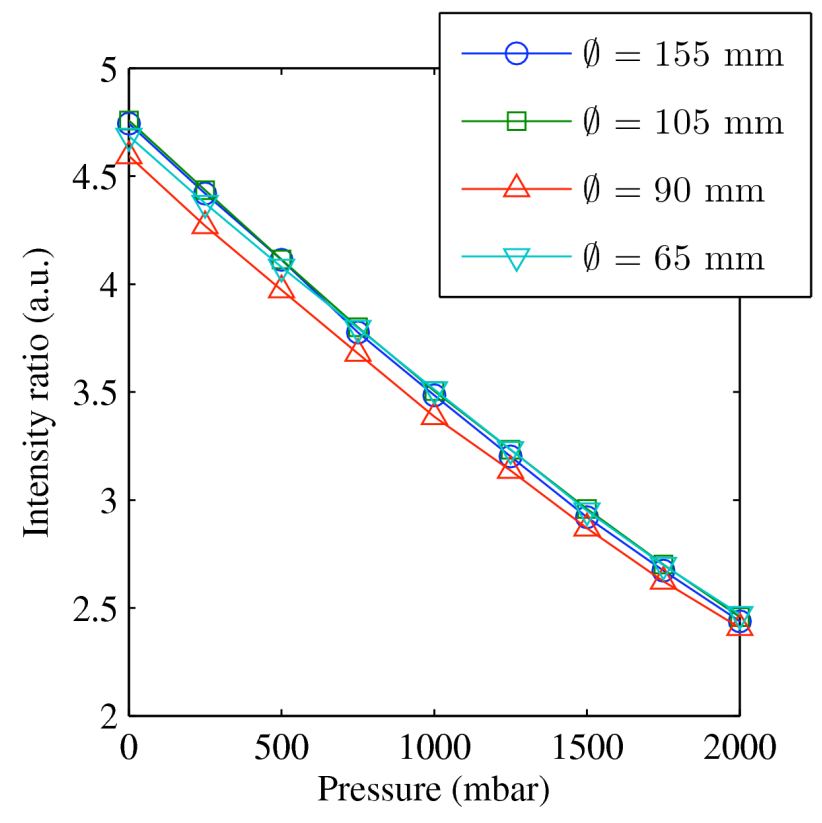

Figure 7: Variation of the ratio between the intensity of the interference peak at $\lambda=670 \mathrm{~nm}$ and the valley at $\lambda=$ $700 \mathrm{~nm}$ as function of relative pressure and for different bending losses

Complementary to the multi-mode FP sensor, the feasibility of applying white light interferometry in the case of a SM fiber sensor is investigated.

In first instance, the SM fiber sensor has been characterized using the previously described solid state laser setup. The average reflected power is experimentally verified to drop $7.8 \% /$ bar (see Fig. 8). However, large measurement errors are observed which have been identified to be mainly contributed by the laser output drift and noise (see Fig. 8, the error bars are representing the standard deviation).

Fig. 9 depicts the white light interference spectra of the SM fiber sensor under test. Despite a more complex interference spectrum due to a more irregular attenuation further away from its central wavelength of $1310 \mathrm{~nm}$, several ascending (e.g. $726 \mathrm{~nm}, 838 \mathrm{~nm}$ ) and descending peaks (e.g. $675 \mathrm{~nm}, 761 \mathrm{~nm}$ ) can still be recognized. This means similar differential techniques can be applied for bending loss compensation in the case of SM fibers by calculating the ratio between an ascending and a descending peak (see Fig. 10). As the SM fiber sensor is designed to work in the infrared region around $1310 \mathrm{~nm}$, one could also investigate the IR spectral behavior of the 
sensor. However, this is beyond the range of the spectrometer used.

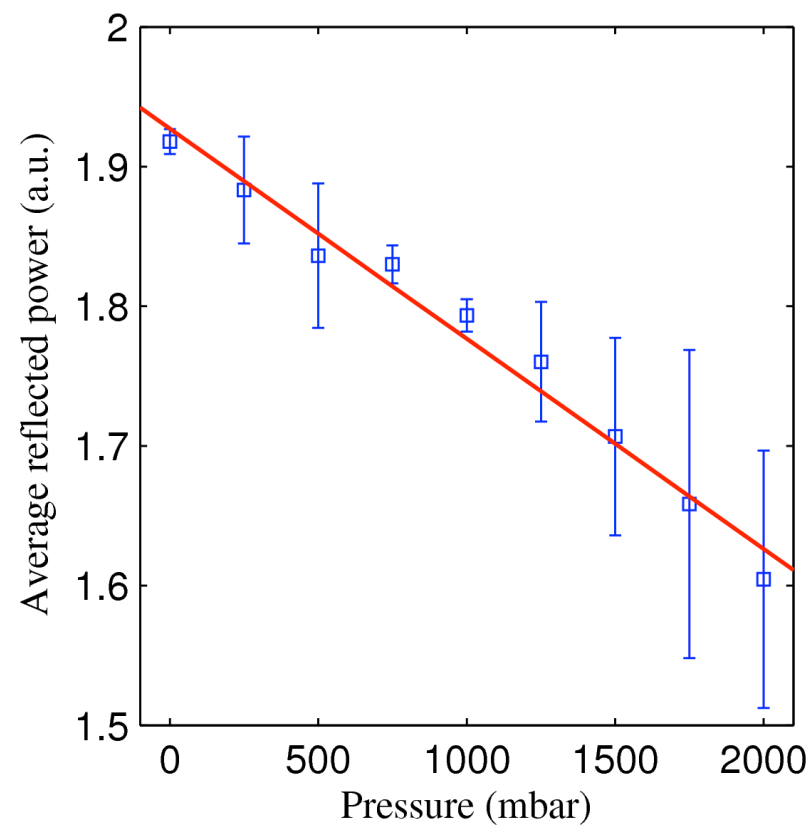

Figure 8: Measured average reflected power as function of applied relative pressure (mbar). Pressure is applied in steps of 250 mbar. $\left(R^{2}=0.9783\right.$, errors bars $=$ std. dev. $)$

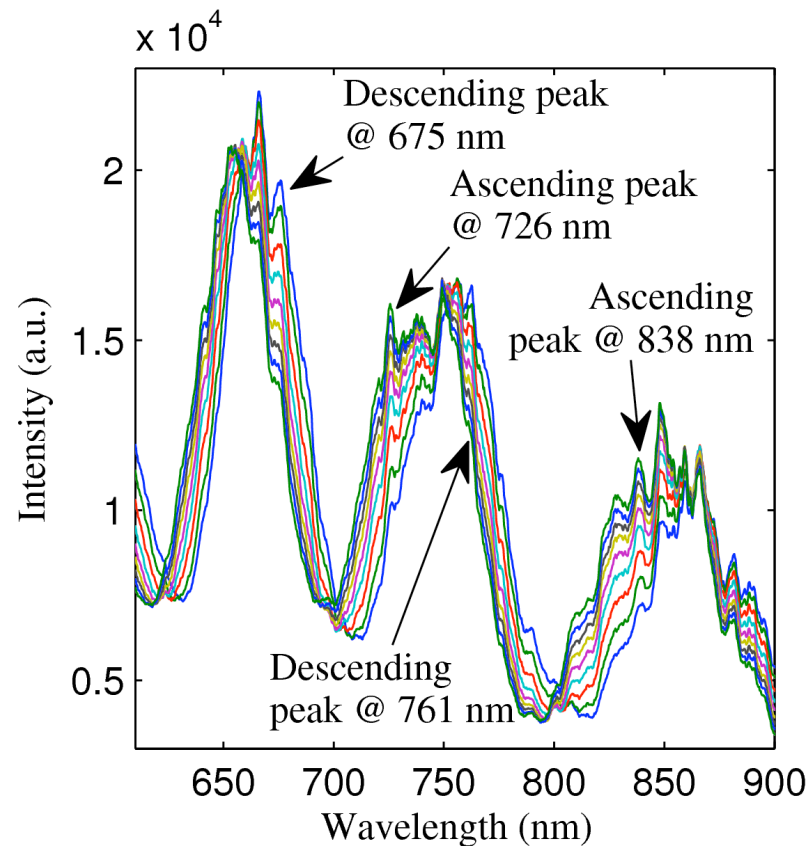

Figure 9: White light interference spectra of SM fiber sensor measured at different relative pressure levels

\section{CONCLUSION}

In conclusion, differential intensity measurements using white light interferometry provide a valid pathway to compensate for bending losses and source fluctuations in ultra-miniature optical fiber sensors. Bending loss sensitivity is demonstrated to be reduced with a factor of $79 \%$. Thus, the proposed differential technique will enable improved rugged high temperature, small size and fiber loss independent pressure sensing.

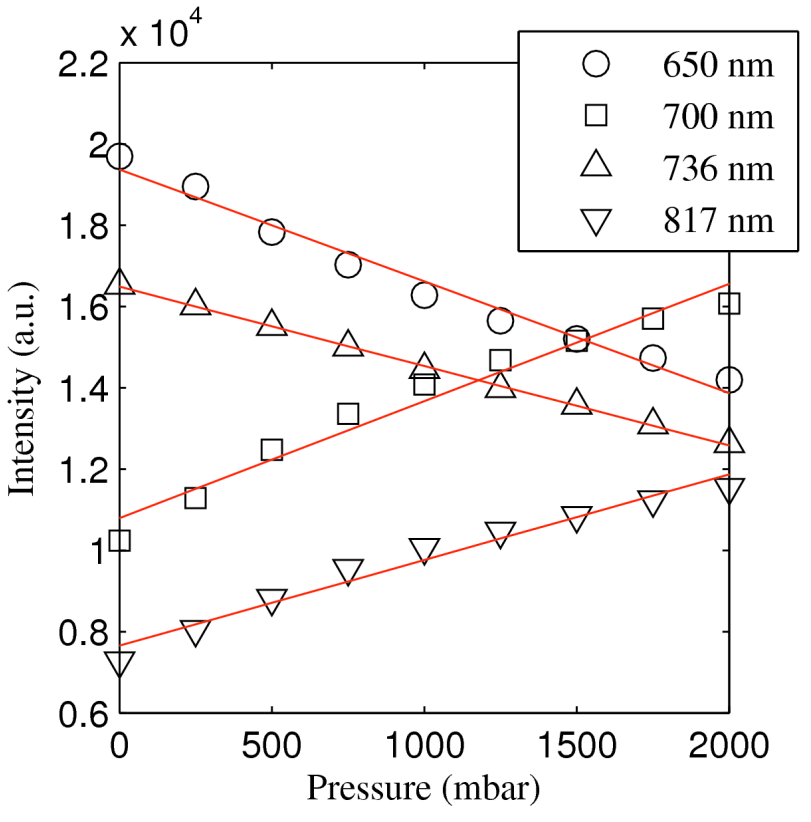

Figure 10: Variation of the intensity as function of the applied pressure for the different interference peaks as indicated in Fig. 9

\section{ACKNOWLEDGEMENTS}

Research funded by a Ph.D. grant of the Agency for Innovation by Science and Technology (IWT).

\section{REFERENCES}

[1] F. Ceyssens, M. Driesen, R. Puers, "An optical absolute pressure sensor for high-temperature applications, fabricated directly on a fiber", $J$. Micromech. Microeng., vol. 19, no. 11, 2009.

[2] A.Wang, H. Xiao, J. Wang, Z. Wang, W. Zhao, R. G. May, "Self-calibrated interferometric-intensity-based optical fiber sensors", J. of Lightwave Techn., vol. 19, pp. 1495-1501, 2001.

[3] K. Totsu, Y. Haga, M. Esashi, "Ultra-miniature fiberoptic pressure sensor using white light interferometry", J. Micromech. Microeng., vol. 15, pp. 71-75, 2005.

[4] J. Yi, E. Lally, A. Wang, Y. Xu, "Demonstration of an all-sapphire Fabry-Pérot cavity for pressure sensing", IEEE Photon. Technol. Lett., vol. 23, no. 1, pp. 9-11, 2011

[5] Y. Zhu, Z. Huang, F. Shen, A. Wang, "Sapphirefiber-based white-light interferometric sensor for high-temperature measurements", Opt. Lett., vol. 30, no. 7,pp. 711-713, 2005

[6] G. Keulemans, F. Ceyssens, R. Puers, "Miniature absolute optical pressure sensor at a fiber tip for high temperature applications", in Proc. Eurosensors XXVI, Kraków, Poland, September 9-12, 2012, pp. 698-701.

\section{CONTACT}

*G. Keulemans, tel: +32-16-321105;

Grim.Keulemans@esat.kuleuven.be 